\title{
EDITORIAL
}

\section{Could resuscitation be based on microcirculation data? No}

\author{
David N. Naumann ${ }^{1,2}$ and Alexandre Lima ${ }^{3 *}$
}

(c) 2018 Springer-Verlag GmbH Germany, part of Springer Nature and ESICM

\section{Introduction}

In modern medical practice, shock resuscitation is usually targeted towards the restoration of conventional parameters, such as those based on blood pressure, acid/ base indicators, and partial pressures of gases. Since these are only surrogate markers of shock, it is appealing to consider the addition of microcirculation data to better inform the physician about the true behaviour of the interface at which shock is most relevant. Recent advances in technology that allow for the acquisition of microcirculatory data, such as transcutaneous oximetry, near-infrared spectroscopy (NIRS), and non-invasive handheld video-microscopy (HVM), make this a tantalising prospect [1]. However, technologies to monitor the microcirculation have been used within the research domain for decades without being adopted into clinical practice. Although some investigators have proposed mechanisms to obtain bedside point-of-care microcirculation data [2-4], these techniques have been controversial, and their uptake slow. In order for microcirculation data to be useful for the resuscitation of patients, data must be easy to acquire at the bedside; easily interpreted, with universal understanding of parameters between clinicians; and must be useful as a target for goal-directed therapy or incorporation into diagnostic criteria. Furthermore, there would need to be widespread adoption of practice, with consensus regarding the utility of microcirculation data. Each of these criteria presents challenges that have the potential to stymie the practice of

\footnotetext{
*Correspondence: a.pintolima@erasmusmc.nl

${ }^{3}$ Department of Intensive Care, Erasmus MC, University Medical Center Rotterdam, Rotterdam, The Netherlands
}

Full author information is available at the end of the article

For contrasting viewpoints, please go to https://doi.org/10.1007/s00134018-5121-0 and https://doi.org/10.1007/s00134-018-5180-2. microcirculatory monitoring before it ever benefits clinical practice.

\section{Problems with acquisition at the bedside}

Although newer technologies such as HVM or NIRS offer a non-invasive approach to acquiring microcirculation data, these techniques require considerable training in order to achieve the highest reproducibility. For example, NIRS measurements rely on vascular occlusion test to estimate microvascular reserve and its use is still controversial in the literature, most likely due to the different methodologies used in different trials [1]. Similarly, in order for videos acquired with HVM to be of any use, the microscopist must minimise movement and pressure artefacts, provide adequate focus and illumination, and take an adequate length of video for analysis [5]. This must also be undertaken in an environment of limited space, movement, and time (Fig. 1). These devices are expensive, meaning that procurement may be more amenable to research grant funding than large-scale clinical provision. Prohibitive costs and training requirements may be insurmountable obstacles to the development of familiarity of these techniques to clinicians, so that they remain niche technologies.

\section{Issues with interpretation}

NIRS data may be confounded by factors other than the true marker of tissue oxygenation and mislead the bedside clinician to assume that hypoperfusion of microcirculation is present, when in fact it may merely reflect cold extremities [2]. Similarly, video clips from HVM must be checked for quality, and then specialist computer software must be employed to derive numerical values for vessel density, perfusion, and heterogeneity [6]. Although automated analysis has been a goal for some years, it is yet to be realised, compounded by delays and frustrating early results. Even if automated analysis is

\section{Springer}




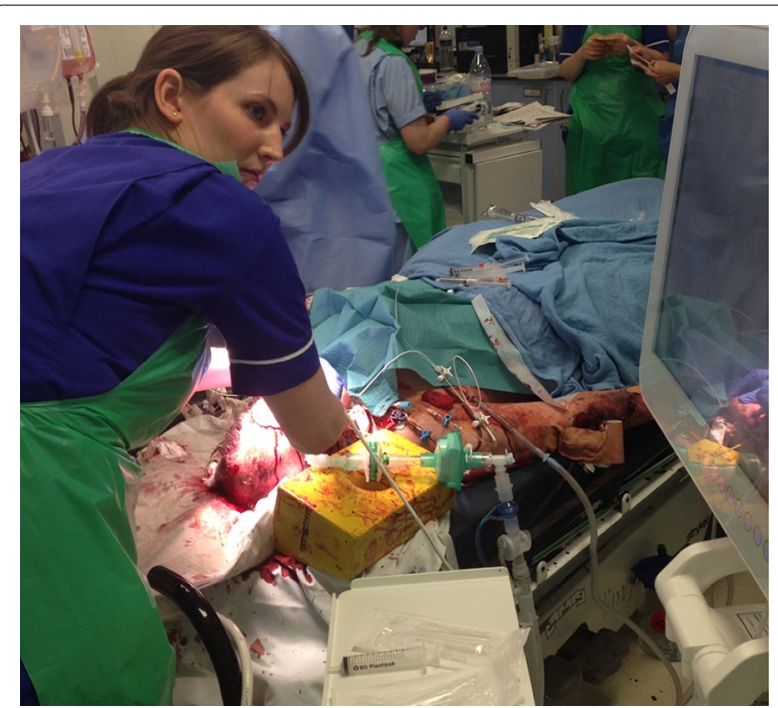

Fig. 1 Bedside sublingual microcirculation monitoring using a handheld video-microscope. This figure illustrates the requirement for space to perform the technique, in a confined environment, compounded by urgency and movement, and surrounded by multiple clinicians involved in the care of the patient

achieved, there are still a limited number of users in the world who are able to make any meaningful interpretation of the parameters of interest. Attempts to propose direct user-interpretation [3, 7] have not led to adoption in clinical practice, since there is still uncertainty about the value of these data and how they might be interpreted during resuscitation. Furthermore, it still remains to be answered whether microcirculation data acquired on one site can mirror equally vulnerable microcirculatory units in other organs.

\section{Pitfalls of guiding therapy}

It is unclear whether the addition of microcirculation data will add any value to the large collection of parameters already available in clinical practice. Modern pointof-care tests and monitoring devices combined with competent clinical examination already provide a plethora of physical, physiological, and biochemical parameters with which to base sound clinical decisions. The addition of a new modality within the scope of resuscitative care must first be shown to improve the accuracy of diagnostic assessment. Although investigators have reported that poor microcirculatory parameters have an association with worse clinical outcomes $[8,9]$, there is uncertainty about whether treatments targeted at microcirculatory parameters have any bearing on clinical outcomes. Within the framework of early goal-directed therapy (EGDT), patients with more favourable microcirculation data have been reported to have superior clinical outcomes in an observational study [10]. However, it is uncertain what role these data may have, especially within the context of increased scepticism about EGDT for patient resuscitation $[11,12]$. There is further uncertainty regarding exactly which treatments might restore the microcirculation during resuscitation, with preclinical data being heterogeneous, and with high risk of bias [13]. For example, which treatments might improve vessel density, perfusion, heterogeneity, or a combination of these? Should microcirculation data to guide resuscitation be used at the end of the macrocirculatory resuscitation process, or guide resuscitation alone? To date, objective data supporting any of these two approaches is merely speculative. In order to overcome the problems with costs, familiarity, and data interpretation, the routine use of microcirculation data for resuscitation will not be achieved without some evidence that the addition of these data improve the decision-making and treatment of patients.

\section{Lack of consensus}

There is no standardized technique for the arterial occlusion test when using NIRS, and there is a lack of supporting evidence regarding which method is superior and more reliable to assess NIRS-derived microcirculatory data $[1,2]$. In the field of microcirculation imaging, the most recent update [14] does not recommend routine clinical use for resuscitation since there is lack of consensus on how this might be achieved, and the definition on the reference values for microcirculation-guided resuscitation end points are not yet established. Furthermore, in their recent consensus on haemodynamic monitoring and circulatory shock, the Task Force of the European Society of Intensive Care Medicine has recommended that techniques to monitor the microcirculation be used for research purposes only (Recommendation 20) [15]. This lack of agreement is based on paucity of evidence for any benefit of using microcirculation data for resuscitation, and a great deal of work is still required if this is ever to be changed. The use of microcirculation data for resuscitation is desirable, and may be achieved in the future, but is not yet justified at the time of writing.

\section{Author details \\ ${ }^{1}$ Academic Department of Military Surgery and Trauma, Royal Centre for Defence Medicine, Queen Elizabeth Hospital, Birmingham, UK. ${ }^{2} \mathrm{NIHR}$ Surgical Reconstruction and Microbiology Research Centre, Queen Elizabeth Hospital, Birmingham, UK. ${ }^{3}$ Department of Intensive Care, Erasmus MC, Uni- versity Medical Center Rotterdam, Rotterdam, The Netherlands.}

\section{Compliance with ethical standards}

Conflicts of interest

The authors declare no conflict of interest. 
Received: 30 January 2018 Accepted: 11 February 2018 Published online: 7 May 2018

\section{References}

1. Lima A (2016) Current status of tissue monitoring in the management of shock. Curr Opin Crit Care 22(3):274-278

2. Lima A, van Bommel J, Sikorska K, van Genderen M, Klijn E, Lesaffre E, Ince C, Bakker J (2011) The relation of near-infrared spectroscopy with changes in peripheral circulation in critically ill patients. Crit Care Med 39(7):1649-1654

3. Naumann DN, Mellis C, Husheer SL, Hopkins P, Bishop J, Midwinter MJ, Hutchings SD (2016) Real-time point of care microcirculatory assessment of shock: design, rationale and application of the point of care microcirculation (POEM) tool. Crit Care 20(1):310

4. Tanaka S, Harrois A, Nicolai C, Flores M, Hamada S, Vicaut E, Duranteau J (2015) Qualitative real-time analysis by nurses of sublingual microcirculation in intensive care unit: the MICRONURSE study. Crit Care 19:388

5. Massey MJ, Larochelle E, Najarro G, Karmacharla A, Arnold R, Trzeciak S, Angus DC, Shapiro NI (2013) The microcirculation image quality score: development and preliminary evaluation of a proposed approach to grading quality of image acquisition for bedside videomicroscopy. J Crit Care 28(6):913-917

6. De Backer D, Hollenberg S, Boerma C, Goedhart P, Buchele G, OspinaTascon G, Dobbe I, Ince C (2007) How to evaluate the microcirculation: report of a round table conference. Crit Care 11(5):R101

7. Arnold RC, Parrillo JE, Phillip Dellinger R, Chansky ME, Shapiro NI, Lundy DJ, Trzeciak S, Hollenberg SM (2009) Point-of-care assessment of microvascular blood flow in critically ill patients. Intensive Care Med 35(10):1761-1766
8. De Backer D, Donadello K, Sakr Y, Ospina-Tascon G, Salgado D, Scolletta S, Vincent JL (2013) Microcirculatory alterations in patients with severe sepsis: impact of time of assessment and relationship with outcome. Crit Care Med 41 (3):791-799

9. Tachon G, Harrois A, Tanaka S, Kato H, Huet O, Pottecher J, Vicaut E, Duranteau J (2014) Microcirculatory alterations in traumatic hemorrhagic shock. Crit Care Med 42(6):1433-1441

10. Trzeciak S, McCoy JV, Phillip Dellinger R, Arnold RC, Rizzuto M, Abate NL, Shapiro NI, Parrillo JE, Hollenberg SM (2008) Early increases in microcirculatory perfusion during protocol-directed resuscitation are associated with reduced multi-organ failure at $24 \mathrm{~h}$ in patients with sepsis. Intensive Care Med 34(12):2210-2217

11. Sharif S, Owen JJ, Upadhye S (2016) The end of early-goal directed therapy? Am J Emerg Med 34(2):292-294

12. Xu JY, Chen QH, Liu SQ, Pan C, Xu XP, Han JB, Xie JF, Huang YZ, Guo FM, Yang $Y$ et al (2016) The effect of early goal-directed therapy on outcome in adult severe sepsis and septic shock patients: a meta-analysis of randomized clinical trials. Anesth Analg 123(2):371-381

13. Naumann DN, Beaven A, Dretzke J, Hutchings S, Midwinter MJ (2016) Searching for the optimal fluid to restore microcirculatory flow dynamics after haemorrhagic shock: a systematic review of preclinical studies. Shock 46(6):609-622

14. Ince C, Boerma EC, Cecconi M et al (2018) Second consensus on the assessment of sublingual microcirculation in critically ill patients: results from a task force of the European Society of Intensive Care Medicine. Intensive Care Med 44(3):281-299

15. Cecconi M, De Backer D, Antonelli M et al (2014) Consensus on circulatory shock and hemodynamic monitoring. Task force of the European Society of Intensive Care Medicine. Intensive Care Med 40(12):1795-1815 